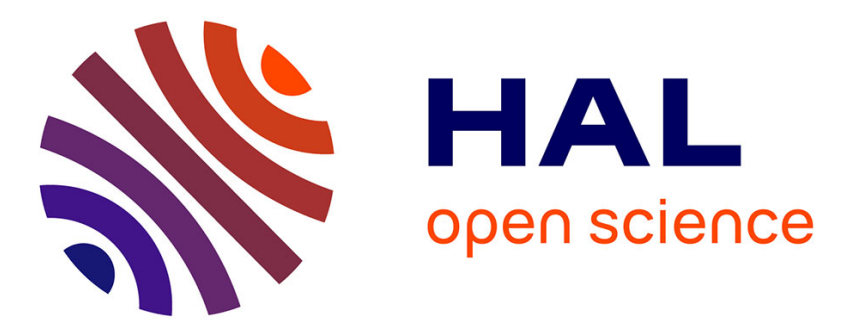

\title{
Nouvel acteur et nouveau phénomène transnationaux : Terre des Hommes et l'adoption internationale (1960-1980)
}

Yves Denéchère

\section{- To cite this version:}

Yves Denéchère. Nouvel acteur et nouveau phénomène transnationaux: Terre des Hommes et l'adoption internationale (1960-1980). Relations internationales, 2010, 142, pp.119-136. halshs01091609

\section{HAL Id: halshs-01091609 \\ https://shs.hal.science/halshs-01091609}

Submitted on 5 Dec 2014

HAL is a multi-disciplinary open access archive for the deposit and dissemination of scientific research documents, whether they are published or not. The documents may come from teaching and research institutions in France or abroad, or from public or private research centers.
L'archive ouverte pluridisciplinaire HAL, est destinée au dépôt et à la diffusion de documents scientifiques de niveau recherche, publiés ou non, émanant des établissements d'enseignement et de recherche français ou étrangers, des laboratoires publics ou privés. 


\section{Nouvel acteur et nouveau phénomène transnationaux : Terre des Hommes et l'adoption internationale (1960-1980)}

La théorie de l'apparition de «nouveaux acteurs » sur la scène mondiale permet d'interroger des phénomènes de l'histoire des relations internationales avec une grille de lecture intéressante mais qui doit s'accompagner d'une approche critique ${ }^{1}$. Le paradigme des nouveaux acteurs est le plus souvent lié à un autre : celui de la transnationalité qui ferait perdre aux Etats la prépondérance que le tropisme réaliste leur accorde dans l'histoire des relations internationales ${ }^{2}$. Au-delà de la théorie - terrain sur lequel l'historien ne peut s'aventurer qu'avec une grande méfiance - l'analyse de certains phénomènes à des moments donnés de l'histoire peut permettre de qualifier tel ou tel acteur et d'appréhender son impact sur l'évolution des relations internationales.

L'adoption internationale peut se prêter à ce genre d'approche. En effet, il s'agit d'un type récent de relations internationales puisqu'il est difficile de le faire remonter au-delà de la Seconde Guerre mondiale, même si des mouvements ont eu lieu dans l'entre-deux-guerres. Cette forme très singulière de migration internationale, qui prend de l'ampleur dans les années 1960, met en interaction des Etats, des particuliers (candidats à l'adoption puis adoptants), des associations, des intermédiaires et des organisations internationales. Chacun de ces protagonistes suit sa propre logique comme l'a bien illustré la question des enfants d'Haïti en cours d'adoption après le séisme de janvier $2010^{3}$.

Dans le grand jeu de l'adoption internationale, l'action du mouvement Terre des Hommes est très particulière sinon unique. Après avoir contribué à développer l'adoption internationale, du milieu des années 1960 au milieu des années 1970, les diverses branches nationales du mouvement sont agitées par un débat qui mène certaines d'entre elles à renoncer à cette forme d'action; c'est le cas de TDH-France. L'étude du positionnement de ce mouvement sur l'adoption internationale et de son évolution en apprend beaucoup au chercheur qui veut saisir la place et le rôle d'un nouvel acteur associatif et la globalité d'un phénomène social transnational inédit.

En Amérique du Nord et en Scandinavie, l'histoire de l'adoption internationale a été explorée dès le début des années 1990, mais pas toujours par des historiens ${ }^{4}$. Le retard de l'historiographie s'explique par le délai d'ouverture des archives concernant un phénomène récent, mais aujourd'hui, des sources sont accessibles. Concernant le rôle que Terre des Hommes a pu jouer dans le développement de l'adoption internationale en partenariat ou en conflit avec les organisations internationales et les Etats, il est possible de s'appuyer sur les archives des ministères français des Affaires étrangères (administration centrale et postes à l'étranger) et des Affaires sociales (et autres ministères assimilés), sur les archives de TDH-F conservées au siège social de l'association et sur des témoignages oraux ${ }^{5}$. Ces sources

\footnotetext{
${ }^{1}$ La théorie de James Rosenau a notamment donné lieu à une approche critique par Samy Cohen : «Les États face aux "nouveaux acteurs" », Politique Internationale, n¹07, printemps 2005, p.409-424.

2 Frank Robert, «Penser historiquement les relations internationales », Annuaire Français de Relations Internationales, Volume IV, 2003.

${ }^{3}$ Sur les différentes logiques développées par les protagonistes on peut se référer à Denéchère Yves, « Pour une histoire de l'adoption internationale en France », Vingtième Siècle Revue d'Histoire, n 102, avril-juin 2009, p. 117-129. Ainsi peut-on distinguer une logique de résultat des candidats à l'adoption, une logique d'action des associations, une logique de régulation des Etats et une logique internationale des droits de l'enfant... Sans oublier les enfants eux-mêmes, acteurs passifs d'un type singulier de relations internationales.

${ }^{4}$ Notamment : Altstein Howard et Simon Rita J. (eds.), Intercountry Adoption : A Multinational Perspective, New York, 1991 ; Zaar Christina, Intercountry Adoptions : What is the State of Research and What New Fields Need to be Investigated?, Stockholm, 1991.

${ }^{5}$ L'auteur exprime sa gratitude aux personnes qui ont témoigné ainsi qu'à Mmes André et Babinault, exprésidente et présidente de Terre des Hommes-France, qui lui ont permis d'accéder aux archives de l'association
} 
permettent également d'aborder la question tout aussi décisive de la manière dont TDH-F définit sa place sur la scène internationale et des éléments qui poussent le mouvement à évoluer vers d'autres formes d'actions que l'adoption.

\section{I - L'apparition de l'adoption et de Terre des Hommes sur la scène internationale}

\section{A - La communauté internationale et les débuts de l'adoption entre pays}

La fin de la Seconde Guerre mondiale a généré en Europe et en Asie des millions de déplacés et réfugiés. Dans ces grandes remues de populations, des enfants perdus, abandonnés, trouvés, survivants ont été pris en charge par des organisations internationales comme le SSI (Service Social International) ou l'UIPE (Union Internationale de Protection de l'Enfance $)^{6}$. L'UNICEF, créée en 1946 pour soutenir les enfants face aux misères de sortie de guerre, ne s'est jamais occupée d'adoption. Dans sa zone d'occupation en Allemagne, la France reçoit de mères allemandes des enfants dont le père est supposé être français. Plusieurs centaines d'entre eux sont adoptés en France dans des circonstances très particulières ${ }^{7}$. Au Japon, des enfants nés de pères américains et de mères japonaises sont abandonnés, le même cas de figure se retrouve lors de la guerre de Corée. Un certain nombre de ces enfants amérasiens font l'objet d'adoptions aux Etats-Unis où Pearl Buck milite pour cette cause, ou en Europe, à l'instar de Joséphine Baker qui en 1954 commence à constituer sa « Tribu Arcen-ciel » en ramenant du Japon deux enfants ${ }^{8}$.

Au cours de l'année 1956, les bureaux du SSI traitent 3500 placements d'enfants dans des familles étrangères; il y a sans doute bien davantage de cas. L'ampleur nouvelle de ce phénomène plus ou moins organisé pousse l'ONU et le Conseil de l'Europe à s'y intéresser'. En 1960, un cycle d'études est organisé à Leysin (Suisse) sous l'égide des Nations Unies. La convention de La Haye «en matière de juridiction, loi applicable et reconnaissance des jugements d'adoption » de 1965 est une réponse à la croissance du nombre des adoptions internationales et du nombre de pays concernés. En 1967, le Conseil de l'Europe - dont le Comité social travaille sur la question depuis le début des années 1960 - édicte une Convention européenne en matière d'adoption des enfants. Fruit d'une réflexion de pays d'accueil riches, elle suscite les critiques des pays-sources du Tiers-monde. La France a une attitude plutôt réservée sur la question de l'adoption entre pays. Dès la fin des années 1940, elle a signifié clairement qu'elle n'encourageait pas (c'est un euphémisme) le départ d'enfants français vers l'étranger à des fins d'adoption. Devenue un pays d'accueil, elle s'appuie sur sa loi de 1966, avec la particularité de l'adoption plénière pratiquement unique au monde, pour ne pas souhaiter de textes internationaux trop contraignants ${ }^{10}$.

\section{B - Terre des Hommes au secours de l'enfance misérable}

et lui ont grandement facilité le travail de recherche. Malheureusement beaucoup d'archives ont été détruites lors d'un incendie.

${ }^{6}$ Olivier Camille, Adopter un enfant, Paris, 1965, p.130-132.

${ }^{7}$ Sur ce cas très particulier : Denéchère Yves, « Des adoptions d'Etat : les enfants de l'occupation française en Allemagne (1945-1952), Revue d'Histoire Moderne et Contemporaine, à paraître en 2010.

${ }^{8}$ Pearl Buck, Children for Adoption, New York, 1964 ; Joséphine Baker adopta douze enfants de couleurs de peau, origines et religions diverses. Toutes les biographies de l'artiste insistent sur sa famille qu'elle a beaucoup médiatisée, y compris dans ses chansons.

${ }^{9}$ Archives du ministère des Affaires étrangères, Conventions administratives et Conventions consulaires (ciaprès CAAC) AC volume 1 carton $\mathrm{n}^{\circ} 3$, «Adoption entre pays : rapport d'un groupe d'experts européens », Nations Unies Genève, 21-25 janvier 1957; comptes-rendus des travaux du comité social du Conseil de l'Europe, 1960.

${ }^{10}$ Denéchère Yves, «La diplomatie française face à la nouveauté des adoptions internationales d'enfants », Revue d'Histoire Diplomatique, 2009, n¹, p.61-75. 
En novembre 1959, lors de l'Assemblée générale de l'ONU, la communauté internationale adopte à l'unanimité la déclaration des Droits de l'enfant. S'appuyant sur les grandes déclarations antérieures relatives aux droits humains, le texte énonce 10 grands principes afin que tout enfant aie « une enfance heureuse et bénéficie, dans son intérêt comme dans l'intérêt de la société, des droits et libertés qui y sont énoncés ${ }^{11}$. Quelques jours auparavant, l'hebdomadaire français La Réforme publiait des reportages sur les camps de "réfugiés» dans l'Algérie en guerre. Le pasteur Jacques Beaumont (président de la Cimade $^{12}$ ) y dénonçait les regroupements de populations opérés par l'armée française et les conditions de vie dramatiques des familles. Un appel à la générosité est lancé afin de venir en aide aux déplacés et surtout aux enfants ${ }^{13}$. La révolte qui le submerge pousse le Suisse Edmond Kaiser (1914-2000) à faire quelque chose pour les enfants qui souffrent, où qu'ils soient dans le monde. Ainsi, à Lausanne, est créé Terre des Hommes : «mouvement de combat et d'intervention immédiate et directe au secours de l'enfance meurtrie ${ }^{14}$. Des enfants algériens sont accueillis en Suisse ; dans plusieurs pays européens, des mouvements Terre des Hommes se créent sur le modèle suisse, ils se fédèrent à Lausanne en 1966.

Au cours de l'été 1962, dans Le Canard Enchaîné, Valentine de Coin-Coin (alias le journaliste Pierre Chatelain-Taillade) lance plusieurs appels en faveur des enfants du Tiersmonde $^{15}$. La mobilisation est au rendez-vous: Terre des Hommes-France est déclarée en décembre. Un article de sa charte précise qu'il faut ramener l'enfant « dans une vie digne de ses droits d'enfant, assuré d'une assistance permanente, tendre et compétente ${ }^{16}$. Dans l'esprit du mouvement, il ne s'agit ni de charité, ni de condescendance, mais de justice. Dès les premières années, des homes sont créés pour assurer la prise en charge d'enfants en Sicile, en Grèce et en Algérie. Une campagne de parrainage est lancée pour financer les opérations en France d'enfants malades du Tiers-monde ; un orphelinat est pris en charge au Cameroun. Ce faisant, à l'instar d'autres mouvements humanitaires créés en Amérique et en Europe - et dès avant la guerre du Biafra, «moment fondateur » de l'humanitaire d'intervention ${ }^{17}$ - le mouvement Terre des Hommes devient un nouvel acteur transnational des relations internationales.

A côté des petits malades que l'on fait venir en Europe pour les soigner, d'autres enfants déshérités, isolés, abandonnés ou orphelins arrivent en Suisse, en Belgique et en France. En effet, la charte de TDH-F précise : «Dans son pays si les circonstance s'y prêtent, ou ailleurs, si tel n'est pas le cas, l'enfant sera nourri, soigné, pourvu de parents valables ». Pourvoir les enfants de parents valables, voilà l'une des missions que s'assigne TDH-F. «L'accueil à vie » conçu par le mouvement se définit ainsi : «l'enfant, jusqu'à sa majorité, conserve son nom, sa nationalité, sa religion d'origine. Ce n'est qu'à 21 ans qu'il lui appartiendra de choisir et d'être adopté si tel est son désir ». Á TDH-F, de détecter ces enfants, de «faire des tractations avec leur gouvernement pour qu'ils nous soient confiés » et de trouver des familles volontaires pour les accueillir en France jusqu'à leur majorité ${ }^{18}$. En fait, et dans le cadre de la loi française sur l'adoption de 1966, presque toutes les familles d'accueil entament très vite une procédure d'adoption plénière de l'enfant accueilli. Se crée ainsi un des premiers grands courants d'adoption internationale vers la France, organisé et promu par TDH-F qui de fait noue des relations avec les Etats de départ et d'accueil. Pour

\footnotetext{
${ }^{11}$ Assemblée générale de l'ONU, 20 novembre 1959.

${ }^{12}$ La Cimade a été fondée en 1939 au sein des mouvements de jeunesse protestants. De sa mission initiale auprès des «évacués » d'Alsace et de Lorraine, elle a conservé son nom (dont la signification d'origine est "Comité Inter-Mouvements Auprès Des Evacués »). Dans les années 1960 elle s'implique auprès des peuples du Sud en lutte pour leur indépendance.

${ }^{13}$ La Réforme, $\mathrm{n}^{\circ} 765,14$ novembre 1959.

${ }^{14}$ Kaiser Edmond, La marche aux enfants, Lausanne, 1979, p.209-222.

${ }^{15}$ Le Canard Enchaîné, «Romance et complainte du retour », 18 juillet 1962, n²178, p.3 ; « Le massacre des innocents », $1^{\text {er }}$ août $1962, n^{\circ} 2180$, p.3.

${ }^{16}$ Archives de Terre des Hommes-France (ci-après TDH-F), historique de Terre des Hommes.

${ }^{17}$ Ryfman Philippe, Une histoire de l'humanitaire, Paris, 2008, p.46 et suivantes.

${ }^{18}$ TDH-F, historique de Terre des Hommes citant une brochure intitulée « 10 ans en France ».
} 
beaucoup de militants tiers-mondistes, pour les congrégations religieuses qui prennent en charge des orphelinats de par le monde, l'adoption est considéré comme un moyen valable parmi d'autres - de venir en aide aux enfants malheureux, de les sauver.

\section{C - Du Vietnam au Biafra en guerre}

Né dans le contexte de la guerre d'Algérie, le mouvement Terre des Hommes fait porter toute son attention sur les pays en guerre, et en premier lieu sur le Vietnam. Dès 1964, TDH-F est agréé par la préfecture du Var «en qualité d'intermédiaire de placement » sur tout le territoire français. En 1967, un enfant algérien et trois enfants vietnamiens sont placés dans des familles en accueil à vie. L'année suivante, ils sont déjà 41 à être extirpés du Vietnam en guerre par TDH-F ; au total, en 1968, le consulat de France à Saigon délivre 92 visas pour des enfants vietnamiens ${ }^{19}$. Marie-Jeanne (Minnie) Galozzi raconte qu'après avoir frôlé la mort de près lors d'une grave maladie et voulant faire quelque chose de sa «deuxième vie », elle rencontre Edmond Kaiser, « un homme hors normes, passionné jusqu'au bout ». La « révolte contre la souffrance des enfants » qu'il exprime en parlant du Vietnam la convainc de s'engager. Elle entre donc à TDH-F et participe à différentes missions et actions au Vietnam $^{20}$. A Saigon, Jeanne Lê Tân, Française mariée à un Vietnamien et employée contractuelle à l'ambassade de France, prend contact avec Terre des Hommes pour « sortir de la misère le plus grand nombre d'enfants possible ${ }^{21}$. A Paris, au début des années 1970, Jean-Pierre Liénasson se rapproche de Terre des Hommes après avoir lu Le massacre des innocents (1970), écrit par Bernard Clavel inspiré par Edmond Kaiser ${ }^{22}$.

A partir de 1968, Terre des Hommes participe à l'évacuation d'enfants du Biafra vers le Gabon. Pris dans la tourmente de la guerre civile qui ravage cette partie du Nigeria depuis 1967, des populations civiles sont victimes de la guerre, de la famine, de la misère. Une nouvelle génération d'humanitaires, notamment des médecins européens emmenés par Bernard Kouchner, réagissent à ce «nouveau Solferino » et inventent une nouvelle forme d'intervention humanitaire ${ }^{23}$. L'Ordre de Malte participe également à l'opération facilitée par le Président Bongo, qui lui-même «adopte» plusieurs enfants biafrais ${ }^{24}$. Arrivés très mal en point à Libreville, remis sur pieds, notamment par une formation médicale des Armées envoyée par la France face à l'urgence sanitaire, des enfants sont envoyés pendant plusieurs mois dans des missions où des religieuses et des coopérants prennent soin d'eux ${ }^{25}$. D'autres sont convoyés vers la Côte d'Ivoire. Les mouvements nationaux Terre des Hommes Allemagne, Danemark, France, Pays-Bas et Suisse inaugurent en novembre 1969 un centre d'hébergement à Mindube (15 km de Libreville). « Malgré les difficultés rencontrées dans le travail de construction, dans l'acheminement des matériaux, du ravitaillement, les enfants arrivent, sont soignés, sont nourris, sont instruits et s'amusent ». Des centaines de Français « parrainent » ces enfants par leurs dons adressés à TDH-F ${ }^{26}$.

\footnotetext{
${ }^{19} \mathrm{CAAC}, \mathrm{AC} 1 \mathrm{n}^{\circ} 6$, dépêche du consul général de France à Saigon au MAE, 27 mars 1969.

${ }^{20}$ Témoignage recueilli par l'auteur, 26 janvier 2010. Minnie Galozzi a été membre de TDH-F de 1968 à 1970 , puis elle a fondé Les Amis des Enfants du Vietnam.

${ }^{21}$ Lê Tân Jeanne, Une forêt de Bambous, 2008, p.39.

${ }^{22}$ Témoignage recueilli par l'auteur, 2 février 2010. Jean-Pierre Liénasson est entré à TDH-F en 1975, il en a été le président de 2001 à 2004.

${ }_{23}$ Rufin Jean-Claude, Le piège, quand l'humanitaire remplace la guerre, Paris, 1986, p.61. Création de Médecins Sans Frontières en 1971.

${ }^{24}$ En 2009, lors de l'accession au pouvoir d'Ali Bongo, la presse internationale s'est fait écho d'une polémique sur ses origines; certains le présentant comme né au Nigeria et l'un des enfants biafrais adoptés par Omar Bongo.

${ }^{25}$ Selon Claudette Nyama, coopérante en 1968-1969 à Oyem (Gabon), mission qui accueille une cinquantaine d'enfants biafrais. Témoignage recueilli par l'auteur, 21 janvier 2010.

${ }^{26}$ TDH-F, historique de Terre des Hommes. En 1969, 736 parrains contribuent chaque mois pour plus de 22000 francs.
} 
Le Quai d'Orsay empêcha toute adoption de ces enfants en France, au motif que «les enfants devaient être maintenus dans leur environnement africain habituel et à proximité de leurs familles », nécessité également rappelée par Caritas Internationalis. En février 1970, la question fait l'objet d'une note interne au Quai d'Orsay qui rappelle la position de la France dans cette affaire et souligne au passage le rôle considéré comme litigieux de l'association Terre des Hommes, «spécialisée dans la pratique des adoptions, mais il a été observé qu'elle ne s'entourait pas toujours des garanties juridiques exigées en France en pareil cas. Aussi bien les initiatives et l'attitude générale de cette organisation ont-elles appelé dès septembre 1968 des réserves ${ }^{27}$. Pour le Biafra au moins, la critique ne semble pas justifiée : nulle part dans les archives de l'association ou dans d'autres fonds on ne trouve trace d'adoptions d'enfants biafrais en France sur le millier que TDH a pris en charge au Gabon. D'ailleurs, la même note précise que seulement «quelques enfants biafrais (trois ou quatre) ont été transférés en France ». En 1971, c'est un député gaulliste, ex-ambassadeur de France au Gabon (19601961) et fondateur du Comité d'action pour le Biafra, qui demandait à son successeur à Libreville de délivrer un visa pour une petite Biafraise de 4 ans qu'il voulait adopter... ${ }^{28}$ Néanmoins, les images d'enfants biafrais ont suscité des velléités d'adoption comme le rapporte Denise Colin, qui commence à s'intéresser à cette question à l'occasion de cet épisode dramatique ${ }^{29}$.

\section{II - Les relations entre Terre des Hommes et les Etats}

Cette question est au cœur de la réflexion sur l'apparition de ce nouvel acteur des relations internationales. Quel type de rapports peut s'établir entre une association de quelques centaines de militants et des Etats reconnus, fussent-ils pauvres et dépendants ? Sans doute faut-il chercher au-delà des rapports de force institués, plutôt du côté d'une relation de fait profitable à l'Etat si elle n'attente pas à sa souveraineté. Les départs d'enfants constituent toujours un risque pour l'Etat car l'opinion est prompte à réagir face à ce qu'elle apparente à un pillage des forces vives du pays. Les politiques menées par les Etats-sources en matière d'adoption internationale s'expliquent en partie ainsi, mais sont également à mettre en parallèle avec les pressions des pays d'accueil et des organisations transnationales et les pratiques internes de l'autorité de l'Etat.

\section{A - Les rapports avec les Etats-sources}

Dès la fin de 1968, face à l'augmentation du nombre de demandes d'enfants et à des départs clandestins, les autorités vietnamiennes s'émeuvent. Elles imposent un délai plus long pour traiter les dossiers et tentent d'empêcher les convois groupés trop visibles et susceptibles de provoquer des réactions hostiles de la population. Une circulaire du gouvernement vietnamien résume bien cet état d'esprit : "Envoyer des orphelins à l'étranger pour y être élevés jusqu'à leur majorité, sans se préoccuper de ce qui leur adviendra ensuite, est non seulement en contradiction avec l'esprit de nos lois, mais peut aussi porter atteinte au prestige national ». La mise en garde semble viser particulièrement Terre des Hommes et son accueil à vie, mais il ne correspond pas à la réalité vécue en France, c'est-à-dire l'adoption plénière dans des délais brefs des enfants accueillis. Toujours est-il que les réticences vietnamiennes entraînent des réclamations des associations. Des candidats à l'adoption saisissent leur député de cette question qui la répercutent au plus haut niveau, comme par exemple William Jacson, député de Meurthe-et-Moselle, qui en 1970 écrit au ministre des Affaires étrangères pour demander l'accélération de la procédure d'adoption. Sollicité, le consul général à Saigon ne

\footnotetext{
${ }^{27} \mathrm{CAAC}$, carton $\mathrm{AC} 1 \mathrm{n}^{\circ} 6$, note pour le cabinet du MAE, 3 février 1970.

${ }^{28}$ Centre des Archives Diplomatiques de Nantes (ci-après CADN), fonds Libreville, carton $\mathrm{n}^{\circ} 100$, lettre du député à l'ambassadeur, 28 janvier 1971.

${ }^{29}$ Témoignage recueilli par l'auteur, 17 février 2010. Denise Colin a été responsable de l'accueil à vie à TDH-F dans les années 1970.
} 
peut que (re)préciser la position du Vietnam et que la France doit restée très prudente la question étant délicate car éminemment politique ${ }^{30}$. D'autant que des expatriés français, comme Jeanne Lê Tân, participent à ce qu'ils considèrent comme un sauvetage d'enfants ${ }^{31}$. En 1973, 53 enfants vietnamiens arrivent en France par TDH-F ${ }^{32}$.

Contrairement à d'autres associations françaises engagées au Vietnam, notamment Les Amis des Enfants du Vietnam ${ }^{33}$, lors de la chute de Saigon en 1975, TDH-F refuse de participer au Baby Lift qui organise en toute hâte le départ d'enfants pris en charge par les humanitaires occidentaux. Présente depuis plusieurs années aussi bien au Vietnam du Nord qu'au Vietnam du Sud, TDH-F fut l'une des rares organisations occidentales à pouvoir continuer à travailler pendant six mois au Vietnam après la victoire des communistes. Quelques années plus tard, par souci de préserver un certain équilibre dans le contexte de la guerre froide, TDH-F décidera de s'engager en Afghanistan après l'invasion de l'armée soviétique, notamment en travaillant avec le Commandant Massoud ${ }^{34}$.

En Corée du Sud, la situation est très différente. Après la guerre qui a figé la division de la péninsule en deux Etats, le nombre d'enfants nés de pères américains et de mères coréennes et abandonnés a posé un grave problème de prise en charge. L'Etat coréen a confié cette mission à une organisation américaine fondée par le pasteur Harry Holt et son épouse ${ }^{35}$. Le Holt Adoption Program (HAP) dispose donc d'un quasi monopole pour le placement d'enfants à l'étranger en vue d'adoption. En 1969, TDH-F est la première œuvre française agréée pour l'adoption d'enfants sud-coréens. Selon 1'ambassadeur de France à Séoul, «les autorités locales paraissent n'élever aucune objection de principe », mais la constitution des dossiers est longue. Le HAP semble débordé par le nombre de demandes d'adoption et avoir des difficultés à organiser matériellement le voyage de ces enfants jusqu'en France ${ }^{36}$.

Une convention, plusieurs fois actualisée, régit les rapports entre TDH-F et HAP, mais les archives montrent des relations parfois tendues, sur le rythme des envois d'enfants, la constitution des dossiers, les aspects financiers, etc. Terre des Hommes doit également composer avec l'Etat sud-coréen. Ainsi, en décembre 1970, le ministère de la Santé et des Affaires sociales de la Corée du Sud ordonne que cesse l'adoption d'enfants vers des pays européens, «en raison de la propagande menée par la Corée du Nord dans le but de ternir le prestige de la république de Corée »; Séoul étant accusé de «vendre les orphelins ». Mais vu sa situation internationale la Corée du Sud n'a pas les moyens de cette politique. Deux mois plus tard, face à «l'opinion mondiale qui s'est élevée » contre sa décision, et aux promesses des consuls occidentaux «de faire tout leur possible» pour empêcher cette situation de se reproduire, le même ministère autorise la reprise des adoptions. Aussitôt, TDH-F demande à l'ambassade de Corée du Sud à Paris l'autorisation de poursuivre son action au rythme de 10 enfants par mois. L'ambassadeur de France à Séoul est mis au courant de cette demande, puis de l'accord reçu des autorités coréennes fin avril $1971^{37}$.

L'impression générale qui se dégage est que l'Etat sud-coréen n'est pas en mesure d'imposer sa politique en matière d'adoption internationale et que l'organisation américaine à qui il a confié toute compétence dans le domaine du secours aux enfants le surpasse. En 1969,

\footnotetext{
${ }^{30} \mathrm{CAAC}, \mathrm{AC} 1 \mathrm{n}^{\circ}$ 6, dépêches du consul général de France à Saigon au MAE, 27 mars 1969 et 14 mars 1970.

${ }^{31}$ Lê Tân Jeanne, op. cit., p.39-50.

${ }^{32}$ CADN, fonds Port-au-Prince, série C, carton n51, TDH-F, « Accueil à vie. Bilan de l'année 1973 ».

${ }^{33}$ Témoignage de Minnie Galozzi-Ullman sur sa participation au Baby Lift et sur l'hésitation des autorités françaises.

${ }^{34}$ TDH-F, CA (conseil d'administration) du 13 avril 1975 ; témoignages de Denise Colin et de Jean-Pierre Liénasson

${ }^{35}$ Sur l'histoire de l'organisation : Holt Children's services inc., The history and the outlook of Holt Children Services Incorporation, Séoul, 1984 ; Maury Françoise, L'adoption interraciale, Paris, 1999, p.34-38.

${ }^{36}$ CAAC, AC 1 n 5, lettre de TDH-F au MAE, 28 avril 1969 ; lettre du MAE à l'ambassadeur de France à Séoul, 8 mai 1969 et réponse du 4 juin 1969.

37 TDH-F, traduction d'une note du ministère de la Santé et des Affaires sociales de Corée du Sud, 27 février 1971 ; courriers de TDH à l'ambassade de Corée du Sud en France (30 mars 1971) et à l'ambassade de France à Séoul (23 avril et 13 mai 1971)
} 
le HAP fait partir 704 enfants, 1083 en 1970,1589 en 1971 et 2014 en $1972^{38}$. Parmi ces enfants, certains ont été pris en charge par TDH-F ( 25 en 1969, 38 en 1970, 93 en 1971 et 103 en 1972) mais cela constitue une très petite minorité et l'association française ne peut traiter d'égal à égal avec l'organisation américaine.

Les rapports entre TDH-F et d'autres Etats-sources sont très divers comme le montrent les exemples du Bangladesh, de la Colombie et d'Haïti. A Dacca, TDH-F a affaire à des religieuses britanniques et bangladaises. En 1973, 10 enfants arrivent en France, mais la situation est constamment délicate. TDH-F n'a aucun renseignement sur les enfants, les pots de vin sont monnaie courante, si l'association les refuse des dossiers restent bloqués. En 1977 le gouvernement met fin à toute adoption vers l'étranger ${ }^{39}$. A Port-au-Prince, le ministre de la Santé, tout en soutenant le projet de TDH-F de créer sur place une crèche pour enfants, affirme à plusieurs reprises «qu'il n'est pas question d'autoriser aucun organisme à "enlever" des enfants d'Haïti ». Néanmoins, en 1973, 14 petits Haïtiens ont été adoptés en France via TDH-F, et bien d'autres dans d'autres pays ${ }^{40}$. Au début des années 1970, Edmond Kaiser critique fortement l'attitude des autorités colombiennes qui selon lui ne gèrent pas correctement les adoptions à l'étranger, s'en suit une tension à l'égard de TDH. Soucieuse de contrôler le sort des orphelins et des enfants abandonnés et de "résoudre ce problème par l'adoption », la Colombie crée en 1972 la Fundación para la Adopción de la Niñez Abandonada (FANA) qui dépend du ministère du Bienestar Familiar. C'est avec cette organisation que TDH-F signe une convention prévoyant le placement de 6 enfants par mois, ce qui nécessite une autorisation préalable de la part de l'Etat colombien demandée à son ambassadeur en France. En 1973, lors d'une mission en Colombie de TDH-F, la FANA se plaint que les Français adoptent trop de filles et pas assez de garçons et de la longueur du délai nécessaire pour l'obtention des visas des enfants partant pour la France. L'association ne peut qu'expliquer que ce sont les autorités françaises qui sont responsables de ces lenteurs, en même temps, elle n'a qu'à se louer du consul de France et de l'attaché d'ambassade à Bogotá qui lui facilitent toutes les démarches auprès des autorités colombiennes... ${ }^{41}$. Il s'agit en fait d'une situation assez courante où TDH-F est prise entre deux feux, entre deux Etats : l'un pays-source et l'autre pays d'accueil.

\section{$B$ - Les rapports avec les autorités françaises}

Comme on a déjà pu le lire, certains représentants de la France à l'étranger émettent des critiques vis-à-vis de l'action de Terre des Hommes, d'autres - plus nombreux semble-t-il - prêtent au contraire leur soutien, officiel et/ou personnel, aux actions entreprises par l'association en faveur des enfants. Les relations entretenues avec les autorités françaises qui ont compétence en matière d'adoption internationale sont d'une toute autre importance pour TDH-F. Outre l'obtention de l'agrément en tant qu'intermédiaire de placement, il s'agit notamment de pouvoir compter sur l'appui de la diplomatie française en cas de difficulté avec des autorités étrangères. Cependant, la logique d'action de l'association et la logique de régulation de 1'Etat ne sont pas toujours conciliables et c'est celle-là qui doit plier devant celle-ci.

L'association se tourne régulièrement vers le Quai d'Orsay pour faire débloquer une procédure, obtenir des visas, etc. A chaque sollicitation, Paris s'assure d'abord de la position

\footnotetext{
${ }^{38}$ Holt Adoption Program, Newsletter, n 8, march-april 1973, « Adoption Placements from Korea 1955-1972», p.9. A la fin de 1972, le HAP avait procédé à plus de 10000 placements d'enfants sud-coréens en vue d'une adoption à l'étranger, dont près de $80 \%$ auprès de couples américains.

39 Témoignage de Denise Colin. TDH-F, lettre de l'équipe de TDH-F à Dacca envoyée au siège, 27 septembre 1977.

${ }^{40} \mathrm{CADN}$, fonds Port-au-Prince C n51, lettre de l'ambassadeur de France en Haïti à TDH-F, 4 avril 1975 ; «Accueil à vie. Bilan de l'année 1973 ».

${ }^{41}$ TDH-F, déclaration de procédure entre TDH-F et FANA, 8 décembre 1972 ; lettre de TDH-F à l'ambassadeur de Colombie à Paris, 21 décembre 1972 ; rapport de mission en Colombie - août 1973.
} 
du gouvernement étranger concerné. Ensuite, il est constamment rappelé à TDH-F la nature de la procédure d'adoption. Par exemple en juillet 1970, l'ambassade de France à Séoul évoque une «affaire purement privée, qui échappe entièrement à la compétence de l'ambassade et qui s'engage sans que celle-ci en soit avertie », les candidats à l'adoption ayant comme interlocuteur TDH-F en rapport avec le HAP. L'ambassade voit donc son rôle limité à la seule délivrance de visas de long séjour, après autorisation du ministère des Affaires étrangères ${ }^{42}$. Au début des années 1970, devant l'ampleur croissante de l'adoption internationale en France - plusieurs centaines d'enfants par an venant essentiellement de foyers principaux mais également en petits nombres de nombreux pays - un groupe interministériel est chargé de proposer des mesures pour réguler le phénomène. Il débute ses travaux en juillet 1971 et immédiatement une prise de conscience semble s'opérer dans les ministères. En août, le Quai d'Orsay répond à un député qui demande qu'on lui indique un intermédiaire : «s'il existe des associations privées qui facilitent les adoptions d'enfants étrangers, il ne m'est pas possible d'engager la responsabilité de mon département en les recommandant ». Alors qu'en janvier de la même année, le même ministère avait répondu à une institutrice demandant la même chose : «le mouvement Terre des Hommes à Le Pradet (Var) devrait être en mesure de faciliter l'adoption par vous-même d'un enfant du Tiersmonde ${ }^{43}$. Il y a donc bien un tournant, les Affaires étrangères ne semblant plus vouloir apparaître comme liées en quoi que ce soit avec des associations comme Terre des Hommes. Ce qui n'empêche pas les membres de TDH-F de continuer à recevoir l'aide de représentants français à l'étranger, tout comme celle, très souvent mentionnée dans les rapports, du personnel d'Air France lors des convoyages des enfants : des directeurs d'agence aux hôtesses de l'air, en passant par les chefs d'escale et les commandants de bord ${ }^{44}$.

Les recommandations issues du groupe de travail interministériel donnent dès 1972 des résultats considérés comme satisfaisants, surtout concernant le consentement à l'adoption des parents ou de l'autorité responsable de l'enfant. De même les avis des Directions Départementales de l'Action Sanitaire et Sociale (DDASS) sur les candidats français à l'adoption sont rendus de manière plus rigoureuse. Mais d'après une correspondance entre le Quai d'Orsay et le ministère de la Santé, cette mesure est mal acceptée par Terre des Hommes qui présenterait encore des avis insuffisants. Sur l'un d'eux, les candidats indiquent qu'ils prendront un enfant «à titre d'essai ». Le Quai d'Orsay prévoit de convoquer le président de TDH-F pour lui indiquer «le caractère irresponsable d'un tel cas de figure ${ }^{45}$. On ne trouve pas trace de cet entretien dans les archives. Peut-être n'a-t-il jamais eu lieu : l'important pour le Quai d'Orsay était surtout de montrer au ministère de la Santé qu'il était désormais mobilisé autant que lui sur le dossier de l'adoption internationale.

En 1975, les relations entre les services de l'Etat et TDH-F sont assez bons pour que ceux-là demandent à certains membres de l'association de jouer un rôle d'experts et de vérificateurs lors de l'arrivée en France d'enfants du Vietnam via le baby lift auquel TDH-F n'a pas participé en tant que telle... Dans cette affaire TDH-F n'est pas un acteur transnational mais a un rôle de régulation que l'Etat lui a délégué. Denise Colin reconnaît dans les familles françaises qui ont obtenu des enfants, certaines qui avaient vu leur dossier refusé par TDH-F, ce qui vient confirmer l'image de sérieux de l'association auprès de l'Etat. Elle refuse de confier des enfants qui déclarent avoir des frères et sœurs et de la famille qui les attend; elle refuse d'en donner un autre à deux hommes qui prétendent agir au nom de la mère adoptive qui est en établissement psychiatrique. Une petite Vietnamienne de 12 ans lui

\footnotetext{
${ }^{42}$ CAAC, AC 1 n$^{\circ} 5$, lettre de TDH-F au MAE, 28 avril 1969 ; lettre du MAE à l'ambassadeur de France à Séoul, 8 mai 1969 et réponse du 4 juin 1969.

${ }^{43} \mathrm{CAAC}, \mathrm{AC} 1 \mathrm{n}^{\circ} 3$, lettre du MAE à un député, 16 août 1971 ; lettre du MAE à une institutrice, 20 janvier 1971.

${ }^{4}$ TDH-F, compte-rendu de voyage en Amérique latine, s.d. (1973 ou 1974); compte-rendu du voyage en Colombie octobre-novembre 1973.

${ }^{45}$ Mission de l'Adoption Internationale sise au Quai d'Orsay (ci-après MAI), carton n 19 , lettre du MAE au ministre de la Santé publique et de la Sécurité sociale, 2 août 1972.
} 
apprend un an après son arrivée qu'elle est en fait la fille d'un responsable de la police qui l'avait fait partir pour la sauver en lui interdisant de révéler la vérité... ${ }^{46}$

\section{III - Motivations éthiques et raisons pratiques du renoncement à l'adoption internationale}

A la fin des années 1970, Terre des Hommes-France renonce aux adoptions internationales et choisit de privilégier d'autres formes d'actions, surtout l'aide au développement directement dans les pays concernés. Ce tournant s'explique donc par «des raisons de philosophie ». La position est désormais celle-ci : «ne faire d'adoption que si Aucune (sic) autre solution n'est possible ${ }^{47}$; comprendre qu'il y en a toujours une ! Plusieurs éléments sont intervenus dans la décision d'arrêter l'activité « accueil à vie ».

\section{A - Les nouvelles données de l'adoption internationale}

Au milieu des années 1970 se produit un tournant bien souligné dans tous les témoignages de responsables d'associations que nous avons recueillis ${ }^{48}$. La chute de Saigon en 1975 entraîne la fermeture du Vietnam. Une des principales sources de l'adoption internationale se trouve tarie. En France, l'inadéquation entre l'offre et la demande est accentuée par les lois votées sous l'impulsion de Simone Veil, ministre de la Santé. La loi sur l'Interruption volontaire de grossesse (IVG) a pu avoir pour effet de diminuer le nombre d'enfants abandonnés adoptables; une loi sur l'adoption de décembre 1976 autorise les couples ayant des enfants légitimes à adopter sans demander une dispense du Président de la République. Une véritable "crise des ciseaux » touche alors l'adoption internationale : diminution de l'offre (intérieure et extérieure) et augmentation de la demande.

Dans ce contexte tendu, les débats autour de l'adoption internationale se font plus aigus, sur fond de problématique Nord/Sud : en un mot, est-elle un bon moyen d'aider au développement? La question de l'enfant et de son bien-être est également posée. Un ouvrage consacré à l'adoption paru en 1976 présente en quelques pages l'action de Terre des Hommes, « considéré généralement comme la plus représentative parmi les intermédiaires de placement d'enfants du Tiers-monde ». Les termes du débat sont clairement exposés : "On a sauvé une vie, c'est ainsi que l'on justifie l'accueil des enfants du Tiers-monde et leur adoption. Je voudrais ajouter: a-t-on rendu heureux un enfant? [...] Nous savons le prix de tout arrachement. Il s'agit donc de poser la question : peut-on, au nom de la solidarité humaine, au nom de la générosité, et a fortiori de motifs moins nobles, déraciner des enfants si d'autres solutions sont possibles ?». Et de proposer le parrainage d'enfants comme alternative plus efficace $^{49}$. Beaucoup d'acteurs de la première époque de l'adoption internationale expriment également la crainte de voir s'organiser des trafics d'enfants, surtout en Amérique latine vers laquelle se tournent de plus en plus de candidats à l'adoption ${ }^{50}$.

Dès 1972, Terre des Hommes-Suisse (TDH-S) a été bousculée par ce débat. Du début de son action à la fin de l'année 1971, 539 enfants étrangers ont été adoptés en Suisse par son intermédiaire. Pour Edmond Kaiser, l'adoption est la «solution totale de la souffrance des enfants orphelins ou abandonnés [...] L'adoption est absolue. Tout autre secours à ces enfants-là est relatif ». Mais beaucoup de membres de TDH-S estiment qu'il faut développer

\footnotetext{
46 Témoignage de Denise Colin.

${ }^{47}$ TDH-F, compte-rendu de la réunion TDH Fédération Internationale sur l'adoption, 9-10 mai 1981 à Bâle.

${ }^{48}$ Notamment Jean Alingrin (CEuvre Emmannuel), 5 mai 2009 ; Jean-Claude Didelot (ASPEL puis Enfants du Mékong), 21 septembre 2009 ; Minnie Galozzi-Ullman (TDH-F puis Amis des Enfants du Vietnam), 26 janvier 2010.

${ }^{49}$ Karila Hélène, L'adopté, enfant roi ou paria, Paris, 1976, p.144-148.

${ }^{50}$ Sur ce point voir : Brigitte Trillat et Sylvia Nabinger, «Adoption internationale et trafic d'enfants : mythes et réalités », in Revue Internationale de Police Criminelle, n428, 1991, pp.18-25.
} 
les autres moyens d'action pour une véritable aide au développement. Edmond Kaiser décide de quitter TDH-S pour (re)fonder Terre des Hommes qui s'installe à Lausanne, d'où une possible confusion entre les associations suisses. TDH-S arrête pratiquement l'adoption internationale, sauf cas exceptionnels, alors que la Fondation Terre des Hommes la pratique et continue à l'encourager. Edmond Kaiser écrit : «Terre des Hommes-France (en attendant les autres) connaîtra les mêmes différends et les mêmes différences $»^{51}$. En effet, toutes les associations nationales ont ce débat, notamment à TDH-Allemagne qui dispose d'une grosse structure gérant l'adoption internationale avec des permanents dont c'est le métier, ce qui introduit une difficulté supplémentaire ${ }^{52}$.

\section{$B$ - Débats sur l'accueil à vie à TDH-F}

Les archives de TDH-F, tant les procès-verbaux des assemblées générales que ceux des séances du conseil d'administration et les comptes-rendus de la commission nationale AAV (accueil à vie), montrent que dès le début des années 1970, des questions se posent concernant l'adoption internationale qui est devenue un véritable phénomène de société. A la fin de l'année 1973 et en quelques années seulement, TDH-F compte 725 accueils à vie ont été réalisés. Deux tendances s'opposent, l'une pour l'adoption internationale comme action de masse permettant de sauver de nombreux enfants ; l'autre pour privilégier d'autres formes d'assistance aux enfants et à leurs familles sur place.

Les conditions de partenariat avec les institutions à l'étranger sont d'abord mises en cause. TDH-F ne participe pas à la sélection des enfants sur place, l'association n'est donc pas sûre que les plus nécessiteux soient envoyés en France ${ }^{53}$. Ainsi, le Holt Chrildren's Services (qui a succédé au HAP et est désormais bien davantage contrôlé par l'Etat coréen) semble presque répondre aux souhaits des candidats à l'adoption en ce qui concerne le type d'enfant attendu; TDH-F craint le développement de l'offre par la demande ${ }^{54}$. Cela renvoie aux questions fondamentales de l'adoptabilité des enfants, de l'attitude qui est tenue face aux parents naturels (surtout face aux mères), du consentement à l'adoption, etc. L'association réclamant des renseignements au Holt, celui-ci se tourne vers des œuvres d'adoption nouvellement créées qui font du placement en France des enfants étrangers leur unique objet. TDH-F ne se reconnaît plus dans ces nouveaux arrivants sur la scène de l'adoption internationale et demande une «enquête approfondie sur les conditions des adoptions réalisées par le Holt ». A la fin de 1977, TDH-F n'envisage l'adoption d'enfants coréens que pour ceux qui sont handicapés ou métissés «dont l'avenir n'est pas rose » en Corée du Sud ${ }^{55}$.

Une deuxième interrogation pèse sur le mode de sélection des familles auxquelles sont confiés (ou non) des enfants : qui fait quoi à TDH-F ? Avec quelle expertise ? En 1973, chaque mois, 100 dossiers d'accueil à vie sont ouverts : 70 passent sans problème, 30 doivent être examinés par une commission ${ }^{56}$. Au siège comme dans les régions, environ trois cents militants travaillent à la sélection des familles. De nombreux documents pointent le besoin de rationaliser les pratiques d'examen des dossiers. Le suivi des enfants après leur placement dans des familles apparaît également insuffisant. Beaucoup d'accueillants ne font pas le rapport demandé dans les semaines qui suivent l'arrivée de l'enfant. En 1975, le conseil d'administration décide de diligenter une enquête sur le statut juridique d'enfants venus en France pour un accueil à vie mais non adoptés. En 1978, un bilan indique que sur 1380 enfants confiés par TDH-F, 1143 ont été adoptés. Sur les 237 non adoptés, 168 sont arrivés

\footnotetext{
${ }^{51}$ Kaiser Edmond, La Marche aux enfants, op. cit., p. 393 et 398.

52 Témoignage de Jean-Pierre Liénasson.

${ }^{53}$ TDH-F, bureau national, 6 juillet 1974.

54 Témoignages de Jean-Pierre Liénasson et de Denise Colin.

${ }^{55}$ TDH-F, CA du 21 mai 1975, des 23 et 24 octobre 1976 et des 5 et 6 novembre 1977.

${ }^{56}$ TDH-F, commission AAV, 30 septembre 1973.
} 
depuis plus de deux ans, bien plus que le temps nécessaire à une procédure d'adoption, ce qui laisse s'interroger sur les intentions des accueillants ${ }^{57}$.

Enfin, quelle attitude tenir en cas d'échec de l'accueil d'un enfant? Toujours d'après le bilan de 1978, 94 enfants ont rencontré des difficultés d'intégration importantes et «ces cas s'aggravent avec le temps »; 42 d'entre eux ont fait l'objet d'une adoption plénière qui interdit tout retour en arrière ; 27 autres ont quitté la famille, rendus volontairement, retirés par TDH-F ou déplacés par l'administration. Une adolescente a disparu depuis 3 ans, une autre a été «rendue » par sa troisième famille d'accueil successive, au regard de son état psychologique l'association doit prendre en charge financièrement son placement en milieu spécialisé $^{58}$. Le retrait d'enfants à des familles est pratiqué par TDH-F qui assume ses responsabilités d'intermédiaire de placement tant que le jugement d'adoption n'est pas rendu.

\section{C-Autour de l'affaire Branco}

Malgré les réserves et les difficultés qui s'amoncellent, lors de l'assemblée générale de Grande Motte (1976), une motion est votée qui affirme : "L'accueil à vie est un moyen comme les autres de sauver les enfants [les membres de TDH-F] demandent qu'il soit poursuivi et développé $» 59$. Mais les débats demeurent virulents et l'ambiance est délétère entre les deux tendances. En 1977, environ 40 \% des adhérents de l'association, privilégiant exclusivement les droits des enfants et soutenant la poursuite et le développement de l'accueil à vie quittent TDH-F. Une grande partie d'entre eux participent à la fondation de Enfance et Partage pour les droits de l'enfant et Accueil et Vie qui développe une activité d'adoption internationale $^{60}$. Lors de son assemblée générale de 1978, TDH-F apporte des modifications à l'orientation de l'accueil à vie qui de fait limitent très fortement ce type d'action. Par exemple, «compte tenu de l'expérience que l'association a des problèmes qui impliquent le choix des enfants, TDH-F se voit dans l'obligation de confier celui-ci à des volontaires spécifiquement sélectionnés pour ce travail ». Il s'agit donc de s'engager fortement sur place sans passer par des intermédiaires. La décision de mener une action d'accueil à vie dans un pays est subordonnée à des études préalables approfondies par des chargés de mission spécifiques, les responsables du secteur géographique, etc. Il est clair que désormais l'accueil à vie ne peut être qu'exceptionnel. Beaucoup de délégations départementales arrêtent toute activité dans ce domaine.

C'est dans ce contexte de renoncement à l'adoption internationale qu'intervient l'affaire Branco qui va précipiter la fin de l'adoption internationale à TDH-F. En mai 1977, au nom de l'association, Denise Colin (responsable AAV), et en raison de mauvais traitements constatés, retire à une famille deux enfants coréens qu'elle lui avait confiés. Ils sont replacés d'abord à plusieurs endroits en France puis en Belgique. L'affaire prend alors une dimension internationale : un procureur belge décide que les enfants qui sont en danger et pas encore français doivent restés en Belgique alors que la justice française exige le retour des enfants en France avant de statuer sur la question des mauvais traitements. Denise Colin subit plusieurs procès, doit se constituer prisonnière pour l'appel à Reims, est menottée (février 1980). Elle est condamnée à neuf mois de prison dont trois mois de prison ferme.

En février 1980, Edmond Kaiser écrit à la responsable AAV de TDH-F afin d'avoir des informations sur l'affaire, car on lui demande «d'étendre à la Suisse un mouvement de solidarité » avec elle. Au passage, il souhaite être informé «de la position claire et définitive de Terre des Hommes-France, et de la vôtre personnellement, dans le domaine de l'adoption ». La réponse est sans ambages : «pour Terre des Hommes-France et moi-même l'adoption reste un moyen tout à fait positif pour des enfants complètement abandonnés sans

\footnotetext{
${ }^{57}$ TDH-F, CA 13 avril 1975 ; PV de l'AG de 1978, annexe 28, p.94.

${ }^{58}$ TDH-F, CA des 18 et 19 octobre 1975 ; PV de l'AG de 1978, annexe 28, p.94.

${ }^{59}$ TDH-F, AG de 1976.

${ }^{60}$ Wolf Maribel, La Colombie écartelée. Le difficile chemin vers la paix, Paris, 2005, 300 p., p.25 ; témoignage de Pierre Anzieu qui fait partie de ceux qui partent vers Accueil et Vie.
} 
foyer ». Mais cette positon est relativisée : «à condition qu'ils ne servent pas les intérêts des uns et des autres, aussi bien sur place dans les pays que chez nous », d'où l'importance de la sélection des enfants et des familles d'accueil ${ }^{61}$. En mars 1980, Edmond Kaiser se dit prêt à venir soutenir Denise Colin lors de son procès en cassation et préconise de prendre contact avec la responsable pour la France du Groupe international de protection des minorités, «car minoritaires sont les victimes ", avec l'espoir d'une éventuelle et peut-être féconde intervention au Parlement européen ${ }^{62}$. C'est un signe intéressant du réseau tissé par Edmond Kaiser et du lobbying dont son organisation est capable pour faire avancer la cause des enfants. En France, les procès de Denise Colin mobilisent les médias, les associations, les députés, l'archevêque de Paris, François Mitterrand... L'élection de ce dernier à la présidence de la République en 1981 permettra à Denise Colin de bénéficier d'une amnistie ; les enfants ne seront jamais rendus et grandiront en Belgique. L'affaire a bien entendu accélerer la fin de l'adoption internationale à TDH-F.

\section{Epilogue}

En juin 1981, à l'instigation de TDH-Suisse qui préconise que les mouvements Terre des Hommes arrête définitivement toute activité d'adoption, une réunion de TDH Fédération Internationale porte sur cette question. Terre des Hommes-France estime le taux d'échecs à $10 \%$ des 1480 adoptions que l'association a permis et rappelle sa philosophie : «ne faire d'adoption que si aucune autre solution n'est possible». Tous les mouvements nationaux présents sont d'accord pour dire que «l'adoption n'est pas le principal objet de leur activité » et qu'il faut « éviter de faire des adoptions en temps de guerre ou de catastrophe naturelle : il est alors trop difficile d'être sûr que l'enfant ne pourra pas un jour retrouver des membres de sa famille ${ }^{63}$. L'Arche de Zoé au Tchad (2007) ou les baptistes américains en Haïti (2010) ne mettront pas en œuvre ce principe de précaution élémentaire.

Dix ans après l'arrêt de son activité adoption internationale, TDH-F mena une grande enquête pour savoir ce qu'étaient devenus « ses » enfants adoptés. Quelques années plus tard, elle donna la parole aux adoptés eux-mêmes dont beaucoup avaient alors plus de vingt ans voire plus de trente ans. Si globalement le bilan peut-être considéré comme positif, les deux enquêtes insistent sur les difficultés d'adaptation et d'intégration des enfants, l'adolescence difficile, les retards scolaires, les questions identitaires, etc. ${ }^{64}$

Aujourd'hui, Terre des Hommes Fédération Internationale est à classer parmi les ONG hostiles à l'adoption internationale qui demandent son encadrement très strict dans le cadre de la convention internationale de La Haye de 1993 et organisent des actions de lobbying auprès des Etats d'accueil. elles estiment que c'est à eux de réguler au maximum la demande afin de ne pas développer l'offre et les abus de toutes sortes ${ }^{65}$. Les Etats sont en même temps confrontés aux pressions des candidats à l'adoption, bien organisés et trouvant aisément des relais politiques et médiatiques. Ils doivent donc déterminer leur politique en matière d'adoption internationale en tenant compte de ces logiques opposées et de bien d'autres variables de leur politique étrangère.

Loin de diminuer le rôle des Etats, en faisant de l'adoption internationale un phénomène de société en France dans les années 1960 et 1970, Terre des Hommes et d'autres mouvements ont suscité une réaction régulatrice de leur part en même temps que des

\footnotetext{
${ }^{61}$ TDH-F, lettre d'Edmond Kaiser à Denise Colin, 12 février 1980 ; lettre de Denise Colin à Edmond Kaiser, 13 mars 1980.

${ }^{62}$ TDH-F, lettres d'Edmond Kaiser à Denise Colin, 25 mars 1980 et $1^{\text {er }}$ avril 1980.

${ }^{63}$ TDH-F, compte-rendu de la réunion TDH Fédération Internationale sur l'adoption, 9-10 mais 1981 à Bâle.

${ }^{64}$ Enquête sur l'adoption internationale. Bilan dix ans après, résultats en 1992 ; Enquête sur l'adoption internationale (II). Les enfants adoptés ont la parole, résultats en 1995.

${ }^{65}$ Adoption: A quel prix? Pour une responsabilité éthique des pays d'accueil dans l'adoption internationale, Etude de Terre des Hommes présentée aux représentants gouvernementaux des pays concernés et aux parlementaires européens, Bruxelles, 26 février 2008.
} 
organisations internationales. Certes, un décalage dans le temps est clairement perceptible : les textes et les législations sont quasi inexistants lorsque débute le phénomène et c'est au moment où certains acteurs transnationaux commencent à s'interroger sérieusement sur les modalités et la finalité de l'adoption internationale que les Etats entreprennent une véritable politique de régulation. Les deux réflexions ont été de pair, se nourrissant l'une l'autre.

Yves Denéchère

Professeur d'histoire contemporaine Université d'Angers - UMR CERHIO 